\title{
DISTRIBUTIONAL TIME-DOMAIN SYSTEM REPRESENTATIONS
}

\author{
Ullrich J. Mönich and Holger Boche
Technische Universität Berlin, Heinrich-Hertz-Chair for Mobile Communications Einsteinufer 25, 10578 Berlin, Germany

\begin{abstract}
In this paper we analyze the convergence behavior of convolutiontype system representations for the Paley-Wiener space $\mathcal{P} \mathcal{W}_{\pi}^{1}$. We completely characterize all stable linear time-invariant (LTI) systems for which we have convergence in the distributional sense by giving a necessary and sufficient condition for convergence. Furthermore, we prove that there are stable LTI systems and signals in $\mathcal{P} \mathcal{W}_{\pi}^{1}$ for which the convolution integral and the convolution sum diverge even in a distributional sense. In signal processing, distributions are often used to show convergence. Surprisingly, here we are in a situation where distributions cannot be used to justify convergence.
\end{abstract}

Index Terms - System representation, convolution, bandlimited signal, distribution, convergence

\section{INTRODUCTION}

The representation of linear time-invariant (LTI) systems by sampling series or convolution integrals is important, not only from a theoretical point of view [1,2], but also for practical applications, where finite sums or integrals can be used for the approximation of the system output. Of course, the convergence behavior of both the sum and the integral is crucial.

Many engineering books [3, 4] give the impression that any LTI system $T$ can be represented as a convolution integral in the form

$$
(T f)(t)=\int_{-\infty}^{\infty} f(\tau) h_{T}(t-\tau) \mathrm{d} \tau,
$$

where $h_{T}$ is the impulse response of the system and $f$ is the input signal. Of course this is true for example for stable LTI systems operating on bandlimited signals with finite energy. However, it is not necessarily true for stable LTI systems acting on other signal spaces. In particular, the behavior for larger spaces, e.g., the PaleyWiener space $\mathcal{P} \mathcal{W}_{\pi}^{1}$, is interesting.

The problem of finding representations of stable LTI systems has been studied for a long time, and several results for spaces of bandlimited signals, which are larger than the space of bandlimited, finite energy signals, have been presented $[2,5,6]$. In [2] Habib derived a convolution integral and a series representation for systems operating on bandlimited signals in the Zakai space [5]. In [6] it has been shown that the integral in (1) is generally not convergent for systems operating on the Paley-Wiener space $\mathcal{P} \mathcal{W}_{\pi}^{1}$.

Although the integral in (1) does not necessarily converge in the classical sense for $\mathcal{P} \mathcal{W}_{\pi}^{1}$, it might be possible that it can still be meaningfully interpreted in a distributional sense. Indeed, distributions can provide a way out of many convergence problems that are present in the classical non-distributional setting. Unfortunately, many engineering textbooks about LTI systems do not treat distributions in a rigorous mathematical manner. Often heuristic arguments prevail.
In this paper we analyze the distributional convergence behavior of the two convolution integrals

$$
\left(A_{N}^{T} f\right)(t):=\int_{-N}^{N} f(\tau) h_{T}(t-\tau) \mathrm{d} \tau
$$

and

$$
\left(B_{N}^{T} f\right)(t):=\int_{-N}^{N} f(t-\tau) h_{T}(\tau) \mathrm{d} \tau,
$$

and the convolution sum

$$
\left(S_{N}^{T} f\right)(t):=\sum_{k=-N}^{N} f(k) h_{T}(t-k)
$$

for signals $f$ in the Paley-Wiener space $\mathcal{P} \mathcal{W}_{\pi}^{1}$ and stable LTI systems $T$. The signal space $\mathcal{P} \mathcal{W}_{\pi}^{1}$ is the largest space in the scale of Paley-Wiener spaces. Thus, the results that are obtained for this space can be seen as an extension of the results for the well-known space $\mathcal{P} \mathcal{W}_{\pi}^{2}$ of bandlimited signals with finite energy. Furthermore, the space $\mathcal{P} \mathcal{W}_{\pi}^{1}$ is important because the convergence behavior of sampling series and convolution integrals for this space is closely related to the mean-square convergence behavior for wide-sense stationary stochastic processes.

We show that the perception that any stable LTI system acting on bandlimited signals can - at least in a distributional setting-be represented as a convolution integral is problematic and not justified in general. Moreover, we completely characterize all stable LTI systems for which the approximation processes (2), (3), and (4) converge to $T f$ for all $f \in \mathcal{P} \mathcal{W}_{\pi}^{1}$ as $N$ tends to infinity.

\section{NOTATION AND DEFINITIONS}

Let $\hat{f}$ denote the Fourier transform of a function $f$, where $\hat{f}$ is to be understood in the distributional sense. $L^{p}(\mathbb{R}), 1 \leq p<\infty$, is the space of all $p$ th-power Lebesgue integrable functions on $\mathbb{R}$, with the usual norm $\|\cdot\|_{p}$, and $L^{\infty}(\mathbb{R})$ is the space of all functions for which the essential supremum norm $\|\cdot\|_{\infty}$ is finite.

For $\sigma>0$ and $1 \leq p \leq \infty$ we denote by $\mathcal{P W}_{\sigma}^{p}$ the Paley-Wiener space of signals $f$ with a representation $f(z)=$ $1 /(2 \pi) \int_{-\sigma}^{\sigma} g(\omega) \mathrm{e}^{i z \omega} \mathrm{d} \omega, z \in \mathbb{C}$, for some $g \in L^{p}[-\sigma, \sigma]$. The norm for $\mathcal{P} \mathcal{W}_{\sigma}^{p}, 1 \leq p<\infty$, is given by $\|f\|_{\mathcal{P} \mathcal{W}_{\sigma}^{p}}=$ $\left(1 /(2 \pi) \int_{-\sigma}^{\sigma}|\hat{f}(\omega)|^{p} \mathrm{~d} \omega\right)^{1 / p}$. For $p=2$ we obtain the Paley-Wiener space $\mathcal{P} \mathcal{W}_{\sigma}^{2}$, which is nothing else than the space of bandlimited signals with finite energy. Moreover, it holds $\|f\|_{\infty} \leq\|f\|_{\mathcal{P} \mathcal{W}_{\sigma}^{p}}$, which implies that every signal in $\mathcal{P} \mathcal{W}_{\sigma}^{p}, 1 \leq p \leq \infty$, is bounded.

We briefly review some facts about stable linear time-invariant (LTI) systems, which will be needed afterwards. A linear system $T: \mathcal{P} \mathcal{W}_{\pi}^{p} \rightarrow \mathcal{P} \mathcal{W}_{\pi}^{p}, 1 \leq p \leq 2$, is called stable if the operator $T$ 
is bounded, i.e., if $\|T\|=\sup _{\|f\|_{\mathcal{P} \mathcal{W}_{\pi}^{p} \leq 1}}\|T f\|_{\mathcal{P} \mathcal{W}_{\pi}^{p}}<\infty$. Furthermore, it is called time-invariant if $(T f(\cdot-a))(t)=(T f)(t-a)$ for all $f \in \mathcal{P} \mathcal{W}_{\pi}^{p}$ and $t, a \in \mathbb{R}$. Note that our definition of stability is with respect to the $\mathcal{P} \mathcal{W}_{\pi}^{p}$-norm and thus is different from the concept of bounded input bounded output (BIBO) stability in general. For every stable LTI system $T: \mathcal{P} \mathcal{W}_{\pi}^{1} \rightarrow \mathcal{P} \mathcal{W}_{\pi}^{1}$ there exists exactly one function $\hat{h}_{T} \in L^{\infty}[-\pi, \pi]$ such that

$$
(T f)(t)=\frac{1}{2 \pi} \int_{-\pi}^{\pi} \hat{f}(\omega) \hat{h}_{T}(\omega) \mathrm{e}^{i \omega t} \mathrm{~d} \omega
$$

for all $f \in \mathcal{P} \mathcal{W}_{\pi}^{1}$. The operator norm of $T$ is given by $\|T\|=$ $\left\|\hat{h}_{T}\right\|_{\infty}$ and the impulse response $h_{T}$ by $h_{T}=T$ sinc. Conversely, every function $\hat{h}_{T} \in L^{\infty}[-\pi, \pi]$ defines a stable LTI system $T$ : $\mathcal{P} \mathcal{W}_{\pi}^{1} \rightarrow \mathcal{P} \mathcal{W}_{\pi}^{1}$. Thus, the space of all stable LTI systems defined on $\mathcal{P} \mathcal{W}_{\pi}^{1}$ is isometrically isomorphic to $L^{\infty}[-\pi, \pi]$. Note that $\hat{h}_{T} \in L^{\infty}[-\pi, \pi] \subset L^{2}[-\pi, \pi]$ and consequently $h_{T} \in \mathcal{P} \mathcal{W}_{\pi}^{2}$. Thus, every stable LTI system on $\mathcal{P} \mathcal{W}_{\pi}^{1}$ has a frequency domain representation according to (5). We will see that a time domain representation in the form (1) is not always possible, even in a distributional setting.

In order to be able to state our key results, we additionally need the concept of distributions. Distributions are continuous linear functionals on some space of test functions. In this paper we deal with test functions in the Schwartz space $\mathcal{S}$, consisting of all continuous functions $\phi: \mathbb{R} \rightarrow \mathbb{C}$ that have continuous derivatives of all orders and fulfill $\sup _{t \in \mathbb{R}}\left|t^{a} \phi^{(b)}(t)\right|<\infty$ for all $a, b \in \mathbb{N}_{0}=\mathbb{N} \cup\{0\}$. $\mathcal{S}^{\prime}$ denotes the dual space of $\mathcal{S}$. We have $\|\phi\|_{\infty}<\infty$ and $\|\phi\|_{1}<\infty$ for all $\phi \in \mathcal{S}$. The Fourier transform maps the space $\mathcal{S}$ onto itself. These properties of $\phi$ will be used extensively in the proofs. For functions $g$, fulfilling $\int_{-\infty}^{\infty}|g(t)|(1+|x|)^{-m}$ for some $m \geq 0$, we can define the linear functional

$$
\phi \mapsto \int_{-\infty}^{\infty} g(t) \phi(t) \mathrm{d} t
$$

on the space $\mathcal{S}$. It can be proven that this functional is continuous and thus defines a distribution [7]. Distributions of the type (6) are called regular distributions. A sequence of distributions $\left\{f_{k}\right\}_{k \in \mathbb{N}}$ in $\mathcal{S}^{\prime}$ is said to converge in $\mathcal{S}^{\prime}$ if for every $\phi \in \mathcal{S}$ the sequence of numbers $\left\{f_{k} \phi\right\}_{k \in \mathbb{N}}$ converges. Thus, a sequence of regular distributions, which is induced by a sequence of functions $\left\{g_{k}\right\}_{k \in \mathbb{N}}$ according to (6), converges in $\mathcal{S}^{\prime}$ if for every $\phi \in \mathcal{S}$ the sequence of numbers $\left\{\int_{-\infty}^{\infty} g_{k}(t) \phi(t) \mathrm{d} t\right\}_{k \in \mathbb{N}}$ converges. For further details about distributions we would like to refer the reader to [7].

\section{CONVOLUTION INTEGRAL}

In this section we analyze the convergence behavior of the two convolution integrals (2) and (3) for stable LTI systems $T$ and signals $f \in \mathcal{P} \mathcal{W}_{\pi}^{1}$.

The theory for stable LTI systems operating on bandlimited signals with finite energy is simple. It is well known that every stable LTI system $T: \mathcal{P} \mathcal{W}_{\pi}^{2} \rightarrow \mathcal{P} \mathcal{W}_{\pi}^{2}$ has the representation

$$
(T f)(t)=\int_{-\infty}^{\infty} f(t-\tau) h_{T}(\tau) \mathrm{d} \tau=\int_{-\infty}^{\infty} f(\tau) h_{T}(t-\tau) \mathrm{d} \tau .
$$

However, (7) is not necessarily true for stable LTI systems operating on signals in $\mathcal{P} \mathcal{W}_{\pi}^{1}$. In [6] it has been shown that the convolution integrals (2) and (3) have a different convergence behavior in general. This is the reason why we treat both convolution integrals in this paper. Moreover, it has been shown that there are stable LTI systems for which the integrals (2) and (3) diverge pointwise. More precisely, for every $t \in \mathbb{R}$ there is a stable LTI systems $T$ such that (2) diverges for some signal $f \in \mathcal{P} \mathcal{W}_{\pi}^{1}$ as $N$ tends to infinity. The same is true for the convolution integral (3).

Although the convolution integrals are not necessarily convergent in the classical (pointwise) sense, it may be possible that (2) and (3), interpreted as a sequence of regular distributions, converge in the distributional sense. If this was true the common conception that every stable LTI system has time-domain representation in the form of a convolution integral would get a rigorous theoretical foundation for the space $\mathcal{P} \mathcal{W}_{\pi}^{1}$, at least in a distributional sense. However, it will turn out that this is not the case.

\subsection{Convergence Behavior of the Convolution Integral I}

Next, we analyze the distributional convergence behavior of the convolution integral (2). For notational convenience, we introduce the abbreviation $A_{N, \phi}^{T} f:=\int_{-\infty}^{\infty}\left(A_{N}^{T} f\right)(t) \phi(t) \mathrm{d} t$.

For the proof of Theorem 1 we need Lemma 1.

Lemma 1. For all stable LTI systems $T, \phi \in \mathcal{S}$, and $N \in \mathbb{N}$ we have

$$
\begin{aligned}
& \sup _{\|f\|_{\mathcal{P} \mathcal{W}_{\pi}^{1}} \leq 1}\left|A_{N, \phi}^{T} f\right| \\
& \quad=\max _{\omega \in[-\pi, \pi]}\left|\frac{1}{\pi} \int_{-\pi}^{\pi} \hat{h}_{T}\left(\omega_{1}\right) \hat{\phi}\left(-\omega_{1}\right) \frac{\sin \left(N\left(\omega-\omega_{1}\right)\right)}{\omega-\omega_{1}} \mathrm{~d} \omega_{1}\right| .
\end{aligned}
$$

Proof. Let $\phi \in \mathcal{S}, N \in \mathbb{N}$, and the stable LTI systems $T$ be arbitrary but fixed. For $f \in \mathcal{P} \mathcal{W}_{\pi}^{1}$ we have

$$
\begin{aligned}
& \int_{-\infty}^{\infty}\left(A_{N}^{T} f\right)(t) \phi(t) \mathrm{d} t=\int_{-\infty}^{\infty} \int_{-N}^{N} f(\tau) h_{T}(t-\tau) \phi(t) \mathrm{d} \tau \mathrm{d} t \\
& =\int_{-\infty}^{\infty} \int_{-N}^{N} \frac{1}{2 \pi} \int_{-\pi}^{\pi} \hat{f}(\omega) \mathrm{e}^{i \omega \tau} h_{T}(t-\tau) \phi(t) \mathrm{d} \omega \mathrm{d} \tau \mathrm{d} t \\
& =\frac{1}{2 \pi} \int_{-\pi}^{\pi} \hat{f}(\omega) \int_{-N}^{N} \mathrm{e}^{i \omega \tau} \int_{-\infty}^{\infty} h_{T}(t-\tau) \phi(t) \mathrm{d} t \mathrm{~d} \tau \mathrm{d} \omega \\
& =\frac{1}{2 \pi} \int_{-\pi}^{\pi} \hat{f}(\omega) \frac{1}{\pi} \int_{-\pi}^{\pi} \hat{h}_{T}\left(\omega_{1}\right) \hat{\phi}\left(-\omega_{1}\right) \frac{\sin \left(N\left(\omega-\omega_{1}\right)\right)}{\omega-\omega_{1}} \mathrm{~d} \omega_{1} \mathrm{~d} \omega
\end{aligned}
$$

where we used Fubini's theorem to interchange the order of the integrals, and the fact that

$$
\int_{-\infty}^{\infty} h_{T}(t-\tau) \phi(t) \mathrm{d} t=\frac{1}{2 \pi} \int_{-\pi}^{\pi} \hat{h}_{T}\left(\omega_{1}\right) \hat{\phi}\left(-\omega_{1}\right) \mathrm{e}^{-i \omega_{1} \tau} \mathrm{d} \omega_{1},
$$

which follows from the generalized Parseval equality. The assertion is a direct consequence of Lemma 17 in [8].

Theorem 1. Let $T$ be a stable LTI system and $\phi \in \mathcal{S}$. Then we have

$$
\lim _{N \rightarrow \infty}\left|A_{N, \phi}^{T} f-\int_{-\infty}^{\infty}(T f)(t) \phi(t) \mathrm{d} t\right|=0
$$

for all $f \in \mathcal{P} \mathcal{W}_{\pi}^{1}$ if and only if there exists a constant $C_{1}=$ $C_{1}(\phi)<\infty$ such that

$$
\max _{\omega \in[-\pi, \pi]}\left|\frac{1}{\pi} \int_{-\pi}^{\pi} \hat{h}_{T}\left(\omega_{1}\right) \hat{\phi}\left(-\omega_{1}\right) \frac{\sin \left(N\left(\omega-\omega_{1}\right)\right)}{\omega-\omega_{1}} \mathrm{~d} \omega_{1}\right| \leq C_{1}(\phi)
$$

for all $N \in \mathbb{N}$. In addition, if (9) is not fulfilled, then there exists a signal $f_{1} \in \mathcal{P} \mathcal{W}_{\pi}^{1}$ such that $\lim \sup _{N \rightarrow \infty}\left|A_{N, \phi}^{T} f_{1}\right|=\infty$. 
Theorem 1 characterizes the convergence of $A_{N}^{T} f$ in $\mathcal{S}^{\prime} . A_{N}^{T} f$ converges to $T f$ in $\mathcal{S}^{\prime}$ for all $f \in \mathcal{P} \mathcal{W}_{\pi}^{1}$ if and only if for all $\phi \in \mathcal{S}$ there exists a constant $C_{1}(\phi)$ such that (9) is fulfilled for all $N \in \mathbb{N}$.

Proof of Theorem 1. First part, " $\Leftarrow$ ": Let $f \in \mathcal{P} \mathcal{W}_{\pi}^{1}, \phi \in \mathcal{S}$, and $\epsilon>0$ be arbitrary but fixed. Since $\mathcal{P} \mathcal{W}_{\pi}^{2}$ is dense in $\mathcal{P} \mathcal{W}_{\pi}^{1}$, there exists a function $f_{\epsilon} \in \mathcal{P} \mathcal{W}_{\pi}^{2}$ with $\left\|f-f_{\epsilon}\right\|_{\mathcal{P} \mathcal{W}_{\pi}^{1}}<\epsilon$. According to Lemma 1 and the assumption (9) we have $A_{N, \phi}^{T}\left(f-f_{\epsilon}\right) \leq \epsilon C_{1}(\phi)$ for all $N \in \mathbb{N}$. Therefore, we obtain

$$
\begin{aligned}
& \left|\int_{-\infty}^{\infty}(T f)(t) \phi(t) \mathrm{d} t-A_{N, \phi}^{T} f\right| \\
& =\mid \int_{-\infty}^{\infty}\left(T\left(f-f_{\epsilon}\right)\right)(t) \phi(t) \mathrm{d} t \\
& \quad+\int_{-\infty}^{\infty}\left(T f_{\epsilon}\right)(t) \phi(t) \mathrm{d} t-A_{N, \phi}^{T} f_{\epsilon}-A_{N, \phi}^{T}\left(f-f_{\epsilon}\right) \mid \\
& <\epsilon\|T\|\|\phi\|_{1}+\left|\int_{-\infty}^{\infty}\left(T f_{\epsilon}\right)(t) \phi(t) \mathrm{d} t-A_{N, \phi}^{T} f_{\epsilon}\right|+\epsilon C_{1}(\phi) .
\end{aligned}
$$

Further, we have $A_{N, \phi}^{T} f_{\epsilon}=\int_{-\infty}^{\infty} \int_{-N}^{N} f_{\epsilon}(t) h_{T}(t-\tau) \mathrm{d} \tau \phi(t) \mathrm{d} t$. Since $f_{\epsilon}, h_{T} \in \mathcal{P} \mathcal{W}_{\pi}^{2}$,

$$
\begin{aligned}
\left|\int_{-N}^{N} f_{\epsilon}(t) h_{T}(t-\tau) \mathrm{d} \tau \phi(t)\right| & \leq \int_{-\infty}^{\infty}\left|f_{\epsilon}(t) h_{T}(t-\tau)\right| \mathrm{d} \tau|\phi(t)| \\
& \leq\left\|f_{\epsilon}\right\|_{2}\left\|h_{T}\right\|_{2}|\phi(t)|
\end{aligned}
$$

and $\phi \in L^{1}(\mathbb{R})$, we can apply Lebesgue's dominated convergence theorem, which, together with (7), leads to $\lim _{N \rightarrow \infty} A_{N, \phi}^{T} f_{\epsilon}=$ $\int_{-\infty}^{\infty}\left(T f_{\epsilon}\right)(t) \phi(t) \mathrm{d} t$. Thus, there is a $N_{0}=N_{0}(\epsilon)$ such that

$$
\left|\int_{-\infty}^{\infty}\left(T f_{\epsilon}\right)(t) \phi(t) \mathrm{d} t-A_{N, \phi}^{T} f_{\epsilon}\right|<\epsilon
$$

for all $N \geq N_{0}(\epsilon)$. Combining (10) and (11), we obtain

$$
\left|\int_{-\infty}^{\infty}(T f)(t) \phi(t) \mathrm{d} t-A_{N, \phi}^{T} f\right|<\epsilon\left(\|T\|\|\phi\|_{1}+C_{1}(\phi)+1\right)
$$

for all $N \geq N_{0}(\epsilon)$. This completes this part of the proof, because $\epsilon>0$ was arbitrary.

Second part, " $\Rightarrow$ ”: Let $\phi \in \mathcal{S}$ be arbitrary but fixed. Since

$$
\left|A_{N, \phi}^{T} f\right| \leq\left|A_{N, \phi}^{T} f-\int_{-\infty}^{\infty}(T f)(t) \phi(t) \mathrm{d} t\right|+\left|\int_{-\infty}^{\infty}(T f)(t) \phi(t) \mathrm{d} t\right|
$$

for all $N \in \mathbb{N}$ and all $f \in \mathcal{P} \mathcal{W}_{\pi}^{1}$, equation (8) and the fact that $\left|\int_{-\infty}^{\infty}(T f)(t) \phi(t) \mathrm{d} t\right| \leq\|T\|\|f\|_{\mathcal{P W}_{\pi}^{1}}\|\phi\|_{1}<\infty$ imply that $\sup _{N \in \mathbb{N}}\left|A_{N, \phi}^{T} f\right|<\infty$ for all $f \in \mathcal{P} \mathcal{W}_{\pi}^{1}$. Hence, by the Banach-Steinhaus theorem [9, p. 98] it follows that $\sup _{N \in \mathbb{N}} \sup _{\|f\|_{\mathcal{P} \mathcal{W}_{\pi}^{1}} \leq 1}\left|A_{N, \phi}^{T} f\right|<\infty$, and consequently

$\sup _{N \in \mathbb{N}} \max _{\omega \in[-\pi, \pi]}\left|\frac{1}{\pi} \int_{-\pi}^{\pi} \hat{h}_{T}\left(\omega_{1}\right) \hat{\phi}\left(-\omega_{1}\right) \frac{\sin \left(N\left(\omega-\omega_{1}\right)\right)}{\omega-\omega_{1}} \mathrm{~d} \omega_{1}\right|<\infty$,

according to Lemma 1, which completes the second part of the proof.

Third part: If (9) is not fulfilled, i.e., if

$\limsup _{N \rightarrow \infty} \max _{\omega \in[-\pi, \pi]}\left|\frac{1}{\pi} \int_{-\pi}^{\pi} \hat{h}_{T}\left(\omega_{1}\right) \hat{\phi}\left(-\omega_{1}\right) \frac{\sin \left(N\left(\omega-\omega_{1}\right)\right)}{\omega-\omega_{1}} \mathrm{~d} \omega_{1}\right|=\infty$,

we have $\sup _{N \in \mathbb{N}} \sup _{\|f\|_{\mathcal{P} \mathcal{W}_{\pi}^{1} \leq 1}}\left|A_{N, \phi}^{T} f\right|=\infty$, according to Lemma 1. Thus, the Banach-Steinhaus theorem implies that there exists a $f_{1} \in \mathcal{P} \mathcal{W}_{\pi}^{1}$ such that $\lim \sup _{N \rightarrow \infty}\left|A_{N, \phi}^{T} f_{1}\right|=\infty$.
In the previous theorem we have seen that if (9) is not fulfilled then there exists a signal $f_{1} \in \mathcal{P} \mathcal{W}_{\pi}^{1}$ such that $A_{N}^{T} f_{1}$ diverges in $\mathcal{S}^{\prime}$. In Theorem 2 we will show that there really exists a stable LTI system for which (9) is not fulfilled, i.e., that there exists a stable LTI system $T_{1}$ such that $A_{N}^{T_{1}} f_{1}$ diverges in $\mathcal{S}^{\prime}$ for some signal $f_{1} \in$ $\mathcal{P} \mathcal{W}_{\pi}^{1}$. In this case, there is not only one divergence creating signal. In fact, the set of signals for which we have divergence is a residual set and therefore is dense in $\mathcal{P} \mathcal{W}_{\pi}^{1}$ [10, p. 12].

Theorem 2. There exists a stable LTI system $T_{1}$ and a signal $f_{1} \in$ $\mathcal{P} \mathcal{W}_{\pi}^{1}$ such that $\lim \sup _{N \rightarrow \infty}\left|A_{N, \phi_{1}}^{T_{1}} f_{1}\right|=\infty$ for some $\phi_{1} \in \mathcal{S}$.

The proof of Theorem 2 is based on the following Theorem, which establishes the connection between the classical convergence of $A_{N}^{T} f$ and the distributional convergence. Theorem 3 is not proved here, because of space constraints.

Theorem 3. Let T be a stable LTI system. The following statements are equivalent.

i) $A_{N}^{T} f$ converges in $\mathcal{S}^{\prime}$ for all $f \in \mathcal{P} \mathcal{W}_{\pi}^{1}$.

ii) $\left(A_{N}^{T} f\right)(t)$ converges pointwise for all $t \in \mathbb{R}$ and all $f \in \mathcal{P} \mathcal{W}_{\pi}^{1}$.

Theorem 3 shows that we do not gain anything regarding the convergence behavior of $A_{N}^{T} f$ for stable LTI systems $T$ and signals $f$ in $\mathcal{P} \mathcal{W}_{\pi}^{1}$ if we consider the more relaxed concept of distributional convergence. If $\left(A_{N}^{T} f\right)(t)$ diverges in the classical (pointwise) sense for some signal $f \in \mathcal{P} \mathcal{W}_{\pi}^{1}$ and some $t \in \mathbb{R}$ then $A_{N}^{T} f$ diverges also in $\mathcal{S}^{\prime}$ for some signal $f \in \mathcal{P} \mathcal{W}_{\pi}^{1}$.

Proof of Theorem 2. According to Theorem 3 in [6], for every $t \in \mathbb{R}$ there exists a stable LTI system $T_{1}$ and a signal $f_{1} \in \mathcal{P} \mathcal{W}_{\pi}^{1}$ such that $\limsup _{N \rightarrow \infty}\left|\left(A_{N}^{T_{1}} f_{1}\right)(t)\right|=\infty$. Thus, the assertion follows from Theorem 3 and Theorem 1 .

Here we see that a convolution type representation of stable LTI systems in the form (2) is not possible in general for the space $\mathcal{P} \mathcal{W}_{\pi}^{1}$, even if the convergence is treated in the distributional sense. In the next section we will see that the same is true for the second convolution integral (3).

\subsection{Convergence Behavior of the Convolution Integral II}

In this section we analyze the second convolution integral (3). Theorem 4 characterizes all stable LTI systems $T$ for which $B_{N}^{T}$ converges in $\mathcal{S}^{\prime}$ to $T f$ for all $f \in \mathcal{P} \mathcal{W}_{\pi}^{1}$. Theorem 4 can be proved analogously to Theorem 1 .

Theorem 4. Let T be a stable LTI system and $\phi \in \mathcal{S}$. Then we have

$$
\lim _{N \rightarrow \infty}\left|B_{N, \phi}^{T} f-\int_{-\infty}^{\infty}(T f)(t) \phi(t) \mathrm{d} t\right|=0
$$

for all $f \in \mathcal{P W}_{\pi}^{1}$ if and only if there exists a constant $C_{2}=$ $C_{2}(\phi)<\infty$ such that

$$
\max _{\omega \in[-\pi, \pi]}\left|\hat{\phi}(-\omega) \frac{1}{\pi} \int_{-\pi}^{\pi} \hat{h}_{T}\left(\omega_{1}\right) \frac{\sin \left(N\left(\omega-\omega_{1}\right)\right)}{\omega-\omega_{1}} \mathrm{~d} \omega_{1}\right| \leq C_{2}(\phi)
$$

for all $N \in \mathbb{N}$. In addition, if (12) is not fulfilled, then there exists a signal $f_{1} \in \mathcal{P} \mathcal{W}_{\pi}^{1}$ such that $\lim \sup _{N \rightarrow \infty}\left|B_{N, \phi}^{T} f_{1}\right|=\infty$. 
Theorem 4 gives a necessary and sufficient condition for the convergence of $B_{N}^{T} f$ in $\mathcal{S}^{\prime} . B_{N}^{T} f$ converges to $T f$ in $\mathcal{S}^{\prime}$ for all $f \in \mathcal{P} \mathcal{W}_{\pi}^{1}$ if and only if for all $\phi \in \mathcal{S}$ there exists a constant $C_{2}(\phi)$ such that (12) is fulfilled for all $N \in \mathbb{N}$.

Using a result from [6], we can show that there exists a stable LTI system $T_{1}$ such that $B_{N}^{T_{1}} f_{1}$ diverges in $\mathcal{S}^{\prime}$ for some $f_{1} \in \mathcal{P} \mathcal{W}_{\pi}^{1}$.

Theorem 5. There exists a stable LTI system $T_{1}$ and a signal $f_{1} \in$ $\mathcal{P} \mathcal{W}_{\pi}^{1}$ such that $\lim \sup _{N \rightarrow \infty}\left|B_{N, \phi_{1}}^{T_{1}} f_{1}\right|=\infty$ for some $\phi_{1} \in \mathcal{S}$.

The proof of Theorem 5 is based on Theorem 6, which relates the classical (uniform) convergence of $B_{N}^{T} f$ and the distributional convergence.

Theorem 6. Let T be a stable LTI system. The following statements are equivalent.

i) $B_{N}^{T} f$ converges in $\mathcal{S}^{\prime}$ for all $f \in \mathcal{P} \mathcal{W}_{\pi}^{1}$.

ii) $B_{N}^{T} f$ converges uniformly on all of $\mathbb{R}$ for all $f \in \mathcal{P} \mathcal{W}_{\pi}^{1}$.

Proof of Theorem 5. According to Corollary 1 in [6] there exists a stable LTI system $T_{1}$ and a signal $f_{1} \in \mathcal{P} \mathcal{W}_{\pi}^{1}$ such that $\lim \sup _{N \rightarrow \infty}\left\|B_{N}^{T_{1}} f_{1}\right\|_{\infty}=\infty$. Thus, the assertion follows from Theorem 6 and Theorem 4.

\section{CONVOLUTION SUM}

The discrete counterpart of the convolution integral (2), which is given by the convolution sum (4) naturally emerges from the finite Shannon sampling series $\left(S_{N} f\right)(t)=\sum_{k=-N}^{N} f(k) \frac{\sin (\pi(t-k))}{\pi(t-k)}$ when some LTI operator $T$ is applied, because

$$
\left(T S_{N} f\right)(t)=\sum_{k=-N}^{N} f(k)\left(T \frac{\sin (\pi(\cdot-k))}{\pi(\cdot-k)}\right)(t)=\left(S_{N}^{T} f\right)(t) .
$$

The sum in (4) is important for practical applications because it uses only the samples $\{f(k)\}_{k \in \mathbb{Z}}$ of the signal $f$. If $\left(S_{N}^{T} f\right)(t)$ converges to $(T f)(t)$ for all $t \in \mathbb{R}$ as $N$ tends to infinity, then $\left(S_{N}^{T} f\right)(t)$ can be used to approximate $(T f)(t)$. Of course the convergence of $\left(S_{N}^{T} f\right)(t)$ is not guaranteed and depends on the signal $f$ and the stable LTI system $T$.

For signals in $\mathcal{P} \mathcal{W}_{\pi}^{2}$ the situation is simple, because we have $(T f)(t)=\sum_{k=-\infty}^{\infty} f(k) h_{T}(t-k)$ for all stable LTI systems $T$ : $\mathcal{P W}_{\pi}^{2} \rightarrow \mathcal{P W}_{\pi}^{2}$ and all signals $f \in \mathcal{P} \mathcal{W}_{\pi}^{2}$. This is due to the convergence of the Shannon sampling series in the $\mathcal{P} \mathcal{W}_{\pi}^{2}$-norm and the continuity and linearity of $T$.

Unfortunately, $\left(S_{N}^{T} f\right)(t)$ does not always converge to $(T f)(t)$ for stable LTI systems operating on $\mathcal{P} \mathcal{W}_{\pi}^{1}$ [11]. Next, we will analyze distributional convergence behavior of (4). For this purpose we introduce the abbreviation $S_{N, \phi}^{T} f=\int_{-\infty}^{\infty}\left(S_{N}^{T} f\right)(t) \phi(t) \mathrm{d} t$.

Theorem 7. Let $T$ be a stable LTI system and $\phi \in \mathcal{S}$. Then we have

$$
\lim _{N \rightarrow \infty}\left|S_{N, \phi}^{T} f-\int_{-\infty}^{\infty}(T f)(t) \phi(t) \mathrm{d} t\right|=0
$$

for all $f \in \mathcal{P} \mathcal{W}_{\pi}^{1}$ if and only if there exists a constant $C_{3}=$ $C_{3}(\phi)<\infty$ such that

$$
\max _{\omega \in[-\pi, \pi]}\left|\frac{1}{2 \pi} \int_{-\pi}^{\pi} \hat{h}_{T}\left(\omega_{1}\right) \hat{\phi}\left(-\omega_{1}\right) \frac{\sin \left[\left(N+\frac{1}{2}\right)\left(\omega-\omega_{1}\right)\right]}{\sin \left(\frac{\omega-\omega_{1}}{2}\right)} \mathrm{d} \omega_{1}\right| \leq C_{3}(\phi)
$$

for all $N \in \mathbb{N}$. In addition, if (13) is not fulfilled, then there exists a signal $f_{1} \in \mathcal{P} \mathcal{W}_{\pi}^{1}$ such that $\lim \sup _{N \rightarrow \infty}\left|S_{N, \phi}^{T} f_{1}\right|=\infty$.
Theorem 7, which can be proved analogously to Theorem 1, completely characterizes all stable LTI systems $T$ for which the convolution sum $S_{N}^{T} f$ converges in $\mathcal{S}^{\prime}$ for all $f \in \mathcal{P} \mathcal{W}_{\pi}^{1}$. In Theorem 9 we will use the characterization of Theorem 7 to show that there exists a stable LTI system $T_{1}$ for which $S_{N}^{T_{1}} f_{1}$ diverges in $\mathcal{S}^{\prime}$ for some $f_{1} \in \mathcal{P} \mathcal{W}_{\pi}^{1}$.

Theorem 8. If $S_{N}^{T} f$ converges in $\mathcal{S}^{\prime}$ for all $f \in \mathcal{P} \mathcal{W}_{\pi}^{1}$ then $\left(S_{N}^{T} f\right)(t)$ converges pointwise for all $t \in \mathbb{R}$ and all $f \in \mathcal{P} \mathcal{W}_{\pi}^{1}$.

Proof. Using the characterization (13) in Theorem 7 for convergence in $\mathcal{S}^{\prime}$ and the characterization for pointwise convergence from [11], the assertion follows after a short calculation.

Theorem 9. There exists a stable LTI system $T_{1}$ and a signal $f_{1} \in$ $\mathcal{P} \mathcal{W}_{\pi}^{1}$ such that $\lim \sup _{N \rightarrow \infty}\left|S_{N, \phi_{1}}^{T_{1}} f_{1}\right|=\infty$ for some $\phi_{1} \in \mathcal{S}$.

Proof. From the results in [11] it can be easily seen that there exists a stable LTI system $T_{1}$ such that $\left(S_{N}^{T_{1}} f_{1}\right)(t)$ diverges for some $t \in \mathbb{R}$ and $f_{1} \in \mathcal{P} \mathcal{W}_{\pi}^{1}$. Thus, the assertion follows from Theorem 8 and Theorem 7 .

We see from Theorem 9 that there are stable LTI systems and signals in $\mathcal{P} \mathcal{W}_{\pi}^{1}$ such that the convolution sum (4) diverges in $\mathcal{S}^{\prime}$. In this regard, we have the same situation as in Section 3, where we analyzed the convolution integral: The divergence of the convolution sum in the classical, non-distributional setting cannot be circumvented by considering the more relaxed concept of distributional convergence. Therefore, a convolution type representation of stable LTI systems in the form (4) is not possible in general for the space $\mathcal{P} \mathcal{W}_{\pi}^{1}$, even if the convergence is treated in the distributional sense.

\section{REFERENCES}

[1] R. L. Stens, "A unified approach to sampling theorems for derivatives and Hilbert transforms," Signal Process., vol. 5, pp. 139-151, 1983.

[2] M. K. Habib, "Digital representations of operators on bandlimited random signals," IEEE Trans. Inf. Theory, vol. 47, no. 1, pp. 173-177, January 2001.

[3] M. Schetzen, Linear Time-Invariant Systems, Wiley \& Sons, 2002.

[4] B. Boulet, Fundamentals of Signals and Systems, Da Vinci Engineering Press, 2005.

[5] M. Zakai, "Band-limited functions and the sampling theorem," Inform. Contr., vol. 8, no. 2, pp. 143-158, 1965.

[6] H. Boche and U. J. Mönich, "Time domain representation of systems on bandlimited signals," in Proc. IEEE Inf. Theory Workshop, May 2008, pp. 51-55.

[7] A. H. Zemanian, Distribution Theory and Transform Analysis, Dover Publications, 1987.

[8] H. Boche and U. J. Mönich, "Limits of signal processing performance under thresholding," Signal Process., vol. 89, no. 8, pp. 1634-1646, August 2009.

[9] W. Rudin, Real and Complex Analysis, McGraw-Hill, 3 edition, 1987.

[10] K. Yosida, Functional Analysis, Springer-Verlag, 1971.

[11] H. Boche and U. J. Mönich, "General behavior of samplingbased signal and system representation," in Proc. IEEE Int. Symp. on Inf. Theory, July 2008, pp. 2439-2443. 